\title{
Risk and risk factors for disability pension among patients with treatment resistant depression- a matched cohort study
}

\author{
Heidi Taipale ${ }^{1,2,3}$, Johan Reutfors ${ }^{4}$, Antti Tanskanen ${ }^{1,2}$, Lena Brandt ${ }^{4}$, Jari Tiihonen ${ }^{1,2}$, Allitia DiBernardo $^{5}$, \\ Ellenor Mittendorfer-Rutz ${ }^{1}$ and Philip Brenner ${ }^{4^{*}}$ (i)
}

\begin{abstract}
Background: Treatment resistant depression (TRD) is common among patients with depression, and is associated with clinical and functional disability. However, the risk and risk factors for being granted disability pension (DP) among patients with TRD have not been investigated.

Methods: All antidepressant initiators in Sweden with a diagnosis of depression in specialized care were identified in nationwide registers 2006-2013 and followed regarding treatment trials. TRD was defined as the start of a third sequential trial. Patients with TRD who were not on DP $(N=3204)$ were matched by age, sex, history of depression, calendar year, and time for treatment start with 3204 comparators with depression and ongoing antidepressant treatment. A proportional Cox Regression was performed with DP as outcome, adjusted for various sociodemographic and clinical covariates.

Results: Compared to the comparison cohort, TRD was associated with a doubled risk for all-cause DP (aHR 2.07; $95 \% \mathrm{Cl} 1.83-2.35)$, DP due to depression $(2.28 ; 1.82-2.85)$ and to any mental disorder $(2.24 ; 1.95-2.57)$ but not due to somatic diagnoses $(1.25 ; 0.84-1.86)$. Among significant risk factors for DP in TRD were female sex, being $>29$ years of age, unemployment and a diagnosis of comorbid personality disorder (ICD-10 codes F60.0-9).

Conclusion: TRD is associated with an elevated risk for DP compared to other patients with depression, with large potential costs for the affected patients and for society. Clinical and therapeutic implications for patients with TRD who are granted DP should be further investigated. Limitation: No clinical data, e.g. type of depression or reason for treatment switch, was available for this study.
\end{abstract}

Keywords: Major depressive disorder, Disability pension, Treatment resistant depression, Epidemiology, Antidepressants

\section{Background}

Depression is a global health problem, affecting about $6 \%$ of the population annually worldwide [1]. The lifetime prevalence is estimated at 16\% [1]. The risk for onset of depression is highest between the ages of 16-43, with a median age of onset at 25 years [2]. Among

\footnotetext{
* Correspondence: philip.brenner@ki.se

${ }^{4}$ Centre for Pharmacoepidemiology, Department of Medicine Solna, Karolinska Institutet, Karolinska University Hospital, Stockholm, Sweden Full list of author information is available at the end of the article
}

adults, depression risk is doubled among women compared to men [3].

By definition, the diagnosis of depression entails significant distress or impairment in social, occupational, or other important areas of functioning [4]. Depression is associated with a number of adverse outcomes for the afflicted individual, such as elevated risk for chronic somatic disease [5], impaired social and physical functioning [6], and death [7]. Furthermore, depression inflicts a large cost on society due to direct and indirect

(C) The Author(s). 2020 Open Access This article is licensed under a Creative Commons Attribution 4.0 International License, which permits use, sharing, adaptation, distribution and reproduction in any medium or format, as long as you give appropriate credit to the original author(s) and the source, provide a link to the Creative Commons licence, and indicate if changes were made. The images or other third party material in this article are included in the article's Creative Commons licence, unless indicated otherwise in a credit line to the material. If material is not included in the article's Creative Commons licence and your intended use is not permitted by statutory regulation or exceeds the permitted use, you will need to obtain permission directly from the copyright holder. To view a copy of this licence, visit http://creativecommons.org/licenses/by/4.0/ The Creative Commons Public Domain Dedication waiver (http://creativecommons.org/publicdomain/zero/1.0/) applies to the data made available in this article, unless otherwise stated in a credit line to the data. 
medical costs [8]. This in combination with high incidence, high risk of recurrence, and a substantial proportion of patients being treatment resistant, depression is one of the leading causes of years lost to disability globally, representing $5 \%$ of the total burden [9].

Outcome studies on depression often focus on symptomatic, rather than functional outcomes, although these do not necessarily correlate [10]. Functional outcomes focus on an individual's recovery in areas such as vocational and social functioning rather than symptom resolution, and are normally not included as outcome parameters in clinical trials. Work disability may be one of the most important functional measures for the individual and for society. It can be measured as temporary, leading to sickness absence, or permanent, in which case the individual may be eligible for disability pension (DP) depending on the national insurance system. Expenditures for DP generally make up a considerable percentage of the total costs for social insurance in welfare states such as Sweden [11]. DP may have large economic and social consequences for the individual, including lower lifetime income and poor quality of life [12]. In a recent meta-analysis including 15 cohort studies, depression was found to increase the risk for DP compared to non-depressed subjects with a pooled risk ratio of 1.82 (95\% confidence interval [CI] 1.45-2.28) among men and 1.62 (95\% CI 1.31-2.02) among women [13].

Treatment-resistant depression (TRD) is most commonly defined as not responding or achieving symptom remission despite two adequate treatment trials [14, 15]. In the second phase of the multicentre European Group for Study of Depression trial, $40 \%$ of patients who initiated antidepressant treatment were affected by TRD [16]. TRD has been associated with an elevated risk of death and suicide [17], and with a higher number of recurrent depressive episodes and hospitalizations [18] compared to depression not classified as treatmentresistant.

TRD has been suggested to be a major contributor to the burden of work disability associated with depression [19]. Depression symptom duration correlates with work disability [20] and the length of depressive episodes seems to predict long-term work disability $[19,21]$. Of depressed patients on sick leave, one third may not return to work within 1 year [22]. Patients who achieve symptom remission show significantly greater improvement in self-rated work functioning compared to those who respond but do not remit [23]. In one clinical study, TRD out-patients incurred higher costs than other depressed patients related to the number of working days lost, and these costs did not correlate with depression severity [24]. Also, a recent re-analysis of data from the Sequenced Treatment Alternatives to Relieve Depression $\left(\mathrm{STAR}^{*} \mathrm{D}\right)$ trial showed greater functional and work impairment as well as lower work productivity among the patients classified with TRD compared to other depressed patients [25].

In clinical studies, there are few reports on long-term consequences for patients with TRD and no studies which report long-term sickness absence or DP [26]. Neither have risk factors for being granted DP among patients with TRD been reported. Among the known risk factors for being granted DP among patients with depression are being female, below 25 or above 45 years of age, low educational level, living alone, residing outside big cities and being born outside Europe, and DP is also associated with antidepressant medication, psychiatric and somatic comorbidities, and long-term sick leave $[27,28]$. In order to fill this gap, pharmacoepidemiological definitions of TRD may be used for research in administrative data. In general, a third claim for an antidepressant drug or other treatment for depression within a specified time frame has been used as a proxy for TRD, an approach that has been used for health economic analyses in US claims data [29-32] as well as in studies in Swedish, British and Taiwanese administrative databases [17, 33, 34].

The aims of this study were (a) to investigate the risk for being granted all-cause and diagnosis-specific DP among patients with TRD compared to other depressed patients with active antidepressant treatment, as a whole and stratified by sex, age, type of living area and history of depression, and (b) to investigate the association between various clinical and sociodemographic factors and DP among patients with TRD.

\section{Methods}

Data sources and study population

This prospective case-cohort study was performed linking data from a combination of nationwide registers in Sweden. Register data is available for researchers after formal application and with a valid ethical permit. Linkage between registers is made through the unique personal identification number assigned to all residents in Sweden, after which data is anonymized before delivery.

The cohort was identified through registers held by the National Board of Health and Welfare. We identified all patients $18-65$ years old with a dispensed prescription of an antidepressant (ATC [Anatomical Therapeutic Chemical]-code N06A), between the years of 2006-2013 in the Prescribed Drug Register (PDR) [35].. The date of the first dispensing in the current episode is hereafter called the index date. The PDR covers data on all dispensed drugs at Swedish pharmacies since July 2005, including drug ATC-code and strength, packet size, date of prescription and dispensing, and also prescriber's text instruction when issued. To identify new treatment episodes, only those patients were selected who had no 
dispensing or recording within 180 days before the index date of a) an antidepressant or any potential add-on medication for treatment of depression according to clinical guidelines (antipsychotics, lithium, lamotrigine, valproate or carbamazepine) [36], and b) administration of ECT (electroconvulsive therapy) or rTMS (repetitive transcranial magnetic stimulation) registered in the National Patient Register (NPR). The NPR includes data on all ICD-10 diagnostic and procedure codes registered during health care contacts in specialized (excluding primary) care, with nationwide in- and out-patient coverage from 1987 and 2001, respectively [37]. Diagnoses in the NPR in general have satisfactory to excellent clinical validity, although e.g. depression has not been specifically validated [37]. Any diagnosis, including depression, is registered by a physician in a health practice setting, in which structured diagnostic interviews for confirmation of diagnoses are common but not mandatory. Patients had to have been residents in Sweden during the full 180 days preceding the dispensing year according to the Total Population Register (TPR), which is a census register held by the government agency Statistics Sweden which contains all individuals resident in Sweden, including immigration and emigration dates and limited demographic information [38]. Further, covariates and outcomes listed in following sections were acquired from the Micro-Data for Analyses of Social insurance (MiDAS) register, held by the National Social Insurance Agency, which includes data on dates and diagnoses of sick leave episodes and DP [39], and sociodemographic data obtained from Statistics Sweden.

In order to identify patients with depression as main treatment indication, we selected those patients who had depression as main diagnosis (ICD-10 codes [International Classification of Diseases, 10th version] F32F33) registered in the NPR within a time interval of 30 days before, and up to 365 days after, the index date. This interval was chosen to capture also patients with initial treatment in primary care which is not covered by the NPR. Patients with any other major psychiatric disorder, defined as a diagnosis of dementia, psychotic or bipolar disorder ever registered in the NPR before the index date, were excluded.

\section{Definition of TRD}

We used a definition of TRD constructed for use in Swedish register data [17]. TRD criteria were met when initiating a third sequential treatment trial for depression after two preceding adequate trials, including the index trial, all within a period of 365 days. The treatment episodes were constructed from data in the PDR regarding dosage, dispensations, package-sizes and prescription texts. An adequate treatment episode was defined as lasting for at least 28 days, and for antidepressants this had to be at least at the lowest defined daily dose for depression according to the ATC-code of the drug. No longer gaps than 28 days were allowed between episodes in order to emulate sequential treatment. Second and third treatment episodes could consist of a) new antidepressant treatment, b) add-on medication with lithium, antipsychotics or anticonvulsants to an existing antidepressant, or c) series of ECT or rTMS. This identified 4377 patients with TRD, of whom 3427 were not on DP at the index date and hence included in the study.

\section{Selection of comparators}

As many factors may affect the association between TRD and DP, we aimed for close socio-demographic and clinical matching between cases and comparators. At the date when TRD criteria were met, each patient with TRD was matched with one comparator - who was not on DP - from the remainder of the depression cohort according to a) age at year of index date ( \pm 3 years), b) sex, c) calendar year of the index prescription, d) type of living area (classified as larger cities, medium-sized municipalities or smaller municipalities), and e) history of a depression diagnosis 1-5 years before index date. The latter was chosen as matching criterion as we hypothesized that it would have substantial impact on the trajectory of antidepressant treatment trials. In order to minimize follow-up bias, comparators also had to have an ongoing 1st or 2nd treatment episode (defined as above through package size and dosing text) to be available for selection. No matching comparators could be found for 223 of the patients with TRD, and they were hence excluded from the study. Cases without comparators were more likely to be male $(54.4 \%$ vs. $42.2 \%$ of cases with comparators) and older (median age 44, IQR 32-56 vs. 36, IQR 25-48) than TRD cases with comparators.

The final sample included $N=3204$ patients with TRD and $\mathrm{N}=3204$ comparators.

\section{Outcomes}

In Sweden, residents aged 19-64 years with a reduced work capacity due to disease or injury can be granted full or part-time DP by the Social Insurance Agency. People aged 19-29 years can be granted temporary DP not only due to work incapacity but also to complete upper-secondary education, and individuals 30-64 years of age can be granted permanent DP. Approximately $1 \%$ of the Swedish population of working age receives DP [11]. The main outcome was granting of DP, part- or full-time, due to any main cause as stated in the MiDAS register.. Secondary outcomes were the first registered occurrence of being granted DP with the main cause diagnosis of depression (ICD-10: F32-33), any mental 
and behavioural disorder (F00-99), or somatic disorder (all causes, except for F00-99).

\section{Covariates}

The analysis also included multiple clinical and sociodemographic covariates. Clinical covariates, measured as diagnoses in in- or outpatient care $<5$ years before index date, were: a) history of anxiety disorders (ICD-10: F40F41), b) history of personality disorders (F60-F61), c) history of other major psychiatric disorder (obsessive compulsive disorders [OCD] F42, eating disorder F50, autism, and hyperactivity disorder F84.0-1, F84.5, F90), d) history of self-harm/suicide attempt (X60-X84, as well as Y10-Y34 [harm with undetermined intent] to avoid underreporting), and e) somatic disorders, expressed as Charlson Comorbidity Index [40] constructed from diagnostic data in the NPR and categorized as 0,1 and $\geq 2$ major comorbidities. In addition, f), substance use disorders (SUD), were categorized in two mutually exclusive categories, alcohol use disorder only, and other/mixed SUD. Alcohol use disorder only included patient with recorded diagnoses of F10.1-F10.9 and/or prescription of disulfiram N07BB01, acamprosate N07BB03, naltrexone N07BB04 or nalmefen N07BB05), but not F11-16, F1819 or prescription of sublingual buprenorphine N07BC01, N07BC51 or methadone N07BC02. If any of the latter diagnoses or prescriptions were present, the patient was counted as other/mixed SUD.

The analyses also included sociodemographic covariates from Statistics Sweden, using the last available data at the index date: a) attained education level $(<9$ years, $10-12$ years, $>12$ years), b) family situation (married/cohabiting, living without children; married/co-habiting, living with children; single, living without children; single, living with children; aged $\leq 20$ years and living with parent/-s), c) country of birth (Sweden/other Europe/ other than Europe). In addition, we assessed previous long-term sickness absence (more than 90 net days on sickness absence during $\leq 365$ days before index date), and employment status during the calendar year preceding the year of index date $(0,1-179$ days, or $\geq 180$ days of registered unemployment).

\section{Statistical analysis}

Complete data were available for the years 2006-2013, which constituted the follow-up period. The follow-up started at the date of fulfilment of TRD definition for patients with TRD and at the corresponding matching date for non-TRD comparators. If a comparator was defined with TRD during the follow-up (i.e. moved from comparator group to TRD group), the follow-up as comparator was censored. The follow-up ended at DP, death, emigration, diagnoses of schizophrenia or bipolar disorder, the end of study follow-up (December 31st, 2013) or if a comparator fulfilled the TRD definition, whichever came first. Proportional Cox regression with TRD status as time-dependent exposure was applied, taking the matched design into account by conditioning on (separate strata) each matched group. All adjusted analyses included all clinical and sociodemographic covariates described above. The main outcome was DP due to any cause, calculating crude and adjusted hazard ratios (aHR) with 95\% confidence intervals (CI) comparing patients with TRD and comparators. For sensitivity, the analysis was also performed stratified on the matching variables. Analyses were also performed with regard to diagnosis-specific DP: due to depressive disorders, any mental or behavioural disorder, and somatic disorders. Risk for DP among patients with TRD compared with comparators was also assessed stratified by each category of matching criteria. Lastly, all covariates were analysed as risk factors for DP separately among patients with TRD. Both unadjusted and adjusted analyses were performed. In the adjusted models all covariates listed above were analysed simultaneously and no variable selection driven from material was performed. Statistical analyses were performed with SAS version 9.3 (SAS Institute, Inc., Cary, NC, USA).

\section{Results}

Table 1 shows descriptive data of the study cohort, which included 3204 persons with TRD and 3204 matched comparators with depression. The majority were women $(58 \%)$ and mean age was 37 years $( \pm S D$ 13). Median follow-up time in the study was somewhat shorter in the TRD cohort than among comparators (1505 days, IQR 494-2105, vs. 1797 days, IQR 669-2238).

During follow-up, 730 patients with TRD and 406 comparators with depression were granted DP, representing incidence rates of DP per 100 persons-years of 6.16 (95\% CI 6.12-6.21) and 3.03 (3.00-3.06), respectively. Crude and adjusted HRs from the full model are shown in Table 2, with the corresponding Kaplan-Meier Curves in Fig. 1. TRD was associated with a doubled risk for all-cause DP compared to comparators (aHR 2.07, 95\% CI 1.83-2.35). The effect was significant over the matching strata (Table 3).

As seen in Table 2, a higher proportion in the TRD group was granted DP due to mental disorders than among comparators ( $85 \%$ vs $76 \%$ ). DP due to depressive disorders were 45 and $43 \%$ of all DPs granted, respectively. The most common diagnostic subclasses of DPs due to mental and behavioural disorders were mood disorders (ICD-10 F3, 51.5\%) and neurotic, stress-related and somatoform disorders (ICD-10 F4, 25.1\%; all frequencies available in Supplemental Table 1). TRD was associated with a significantly increased risk of DP due 
Table 1 Characteristics of patients with treatment resistant depression (TRD) vs matched comparators ${ }^{\mathrm{a}}$ with treatment for depression

\begin{tabular}{|c|c|c|c|}
\hline & $\begin{array}{l}\text { TRD } \\
N=3204\end{array}$ & $\begin{array}{l}\text { Comparators } \\
N=3204\end{array}$ & $p$ \\
\hline \multicolumn{4}{|l|}{ n (\%) } \\
\hline Men & $1363(42.5)$ & $1363(42.5)$ & matched \\
\hline Women & $1841(57.5)$ & $1841(57.5)$ & \\
\hline \multicolumn{4}{|l|}{ Age (years) $^{b}$} \\
\hline $18-29$ & 1245 (38.9) & $1251(39.0)$ & matched \\
\hline $30-49$ & 1333 (41.6) & $1328(41.5)$ & \\
\hline $50-69$ & $626(19.5)$ & $625(19.5)$ & \\
\hline Type of living area & & & matched \\
\hline Small municipality & $842(26.3)$ & $842(26.3)$ & \\
\hline $\begin{array}{l}\text { Medium-sized } \\
\text { municipality }\end{array}$ & 1109 (34.6) & 1109 (34.6) & \\
\hline Large city & $1253(39.1)$ & $1253(39.1)$ & \\
\hline $\begin{array}{l}\text { Attained education } \\
\text { level }^{b}\end{array}$ & & & 0.0017 \\
\hline$\leq 9$ years & $969(30.2)$ & $842(26.3)$ & \\
\hline 10-12 years & $1442(45.0)$ & $1504(46.9)$ & \\
\hline$\geq 13$ years & $793(24.8)$ & $858(26.8)$ & \\
\hline Family situation $^{b}$ & & & 0.0942 \\
\hline $\begin{array}{l}\text { Married, living } \\
\text { without children }\end{array}$ & $296(9.2)$ & $310(9.7)$ & \\
\hline $\begin{array}{l}\text { Married, living with } \\
\text { children }\end{array}$ & $768(24.0)$ & $695(21.7)$ & \\
\hline $\begin{array}{l}\text { Unmarried, living } \\
\text { without children }\end{array}$ & $1419(44.3)$ & $1508(47.1)$ & \\
\hline $\begin{array}{l}\text { Unmarried, living } \\
\text { with children }\end{array}$ & $327(10.2)$ & $299(9.3)$ & \\
\hline $\begin{array}{l}\leq 20 \text { years, living } \\
\text { with parent/-s }\end{array}$ & $394(12.3)$ & $392(12.2)$ & \\
\hline Country of birth & & & $<0.0001$ \\
\hline Sweden & $2420(75.5)$ & 2647 (82.6) & \\
\hline $\begin{array}{l}\text { Other European } \\
\text { country }\end{array}$ & $165(5.2)$ & $123(3.8)$ & \\
\hline Other than Europe & 619 (19.3) & 434 (13.6) & \\
\hline $\begin{array}{l}\text { Long-term sickness } \\
\text { absence }^{c}\end{array}$ & $429(13.4)$ & $418(13.1)$ & 0.6849 \\
\hline Unemployment $^{d}$ & & & 0.2803 \\
\hline $1-179$ days & $643(20.1)$ & $620(19.4)$ & \\
\hline$\geq 180$ days & $185(5.8)$ & $161(5.0)$ & \\
\hline $\begin{array}{l}\text { History of depression } \\
\text { diagnosis }\end{array}$ & $536(16.7)$ & $536(16.7)$ & matched \\
\hline $\begin{array}{l}\text { History of anxiety } \\
\text { disorder }^{\mathrm{e}}\end{array}$ & $621(19.4)$ & $638(19.9)$ & 0.5930 \\
\hline $\begin{array}{l}\text { History of personality } \\
\text { disorder }^{\mathrm{e}}\end{array}$ & $97(3.0)$ & $87(2.7)$ & 0.4544 \\
\hline $\begin{array}{l}\text { History of substance } \\
\text { use disorder }\end{array}$ & & & 0.0425 \\
\hline
\end{tabular}

Table 1 Characteristics of patients with treatment resistant depression (TRD) vs matched comparators ${ }^{\mathrm{a}}$ with treatment for depression (Continued)

\begin{tabular}{llll}
\hline & TRD & Comparators & $p$ \\
& $N=3204$ & $N=3204$ & \\
\hline None & $2895(90.4)$ & $2847(88.9)$ & \\
Alcohol only & $143(4.5)$ & $187(5.8)$ & \\
$\begin{array}{l}\text { Other/mixed } \\
\text { History of other } \\
\text { psychiatric disorder }\end{array}$ & $166(5.2)$ & $170(5.3)$ & \\
$\begin{array}{l}\text { History of self harm/ } \\
\text { suicide attempt }\end{array}$ & $190(5.9)$ & $195(6.1)$ & 0.7927 \\
CCl $^{\mathrm{e}}$ f & $208(6.5)$ & $277(7.1)$ & 0.3454 \\
0 & & & \\
1 & $3029(94.5)$ & $3016(94.1)$ & \\
$\geq 2$ & $143(4.5)$ & $155(4.8)$ & \\
\hline
\end{tabular}

${ }^{a}$ Comparators matched on age, sex, calendar year of the index prescription, history of depression, and size of living area

${ }^{\mathrm{b}}$ At time of first antidepressant prescription

c > 90 net days in the 365 days preceding first antidepressant prescription

${ }^{d}$ In the year preceding first antidepressant prescription

${ }^{e}$ Within 5 years before first antidepressant prescription

${ }^{f}$ Charlson's comorbidity index [40]

to depressive disorders (aHR 2.28; 95\% CI 1.82-2.85) and DP due to any mental and behavioural disorder (aHR 2.24, 95\% CI 1.95-2.57) but not due to somatic disorders (aHR 1.25, 95\% CI 0.84-1.86)..

In the risk factor analysis for DP among patients with TRD (Table 4), significant sociodemographic risk factors were: being female compared to male (aHR 1.21, 95\% CI $1.03-1.41$ ), older age compared to $18-29$ years old (5069 years old: aHR 1.70, 95\% CI 1.32-2.19), being born outside of Europe compared to in Sweden (aHR 1.55, 95\%CI 1.29-1.85), long-term sickness absence (aHR 3.26, 95\% CI 2.74-3.87) and being unemployed (aHR 1.24, 95\%CI 1.03-1.50). Regarding family status, being $(\leq 20 \mathrm{y})$ and living with parents inferred the highest risk (aHR 1.90, 95\% CI 1.30-2.78) and living in a single household with children the lowest risk (aHR 0.66, 95\% CI 0.46-0.94), compared to being married/cohabitant without children (ref). Significant clinical risk factors were having a diagnosis of personality disorder (aHR $1.58,95 \%$ CI $1.05-2.38$ ) and CCI 1 or above (CCI 1: aHR $1.41,95 \%$ CI 1.03-1.92).

\section{Discussion}

The main finding of this study was that patients with TRD had a doubled risk for being granted DP compared to other patients with depression and ongoing treatment. The risk was similar among patients with a first-time depression diagnosis compared to those with recurrent depression. The risk was increased for DP granted due to a depressive disorder or any mental disorder, but not for somatic diagnoses. Among patients with TRD, being 
Table 2 Risk for disability pension (DP) among patients with treatment-resistant depression (TRD) vs. matched comparators with treatment for depression. Unadjusted and adjusted hazard ratios (HR) with 95\% confidence intervals (Cl) for all-cause and causespecific DP

\begin{tabular}{|c|c|c|c|}
\hline Outcome & $\mathrm{N}$ (\% of DPs granted) & Unadjusted HR (95\% Cl) & Adjusted HR $(95 \% \mathrm{Cl})^{\mathbf{b}}$ \\
\hline \multicolumn{4}{|l|}{ All-cause DP } \\
\hline Comparators $^{\mathbf{a}}(n=3204)$ & $406(100)$ & Ref & Ref \\
\hline $\operatorname{TRD}(n=3204)$ & $730(100)$ & $1.90(1.72-2.11)$ & $2.07(1.83-2.35)$ \\
\hline \multicolumn{4}{|c|}{ DP due to mental and behavioural disorders } \\
\hline Comparators & $309(76.1)$ & Ref & Ref \\
\hline TRD & $621(85.1)$ & $2.13(1.89-2.39)$ & $2.24(1.95-2.57)$ \\
\hline \multicolumn{4}{|c|}{ Of which DP due to depression (F32-33) } \\
\hline Comparators & $134(43.4)$ & Ref & Ref \\
\hline TRD & $277(44.6)$ & $2.15(1.80-2.56)$ & $2.28(1.82-2.85)$ \\
\hline \multicolumn{4}{|l|}{ DP due to somatic disorders } \\
\hline Comparators & $97(23.9)$ & Ref & Ref \\
\hline TRD & $109(14.9)$ & $1.22(0.98-1.51)$ & $1.25(0.84-1.86)$ \\
\hline
\end{tabular}

${ }^{a}$ Comparators matched on age, sex, calendar year of the index prescription, history of depression, and size of living area

${ }^{\mathrm{b}}$ Adjusted for attained educational level, marital/ parental status, country of birth, long-term sickness absence, unemployment, anxiety, personality and other psychiatric disorders, substance use disorder, history of self-harm/suicide attempt and Charlson's Comorbidity index

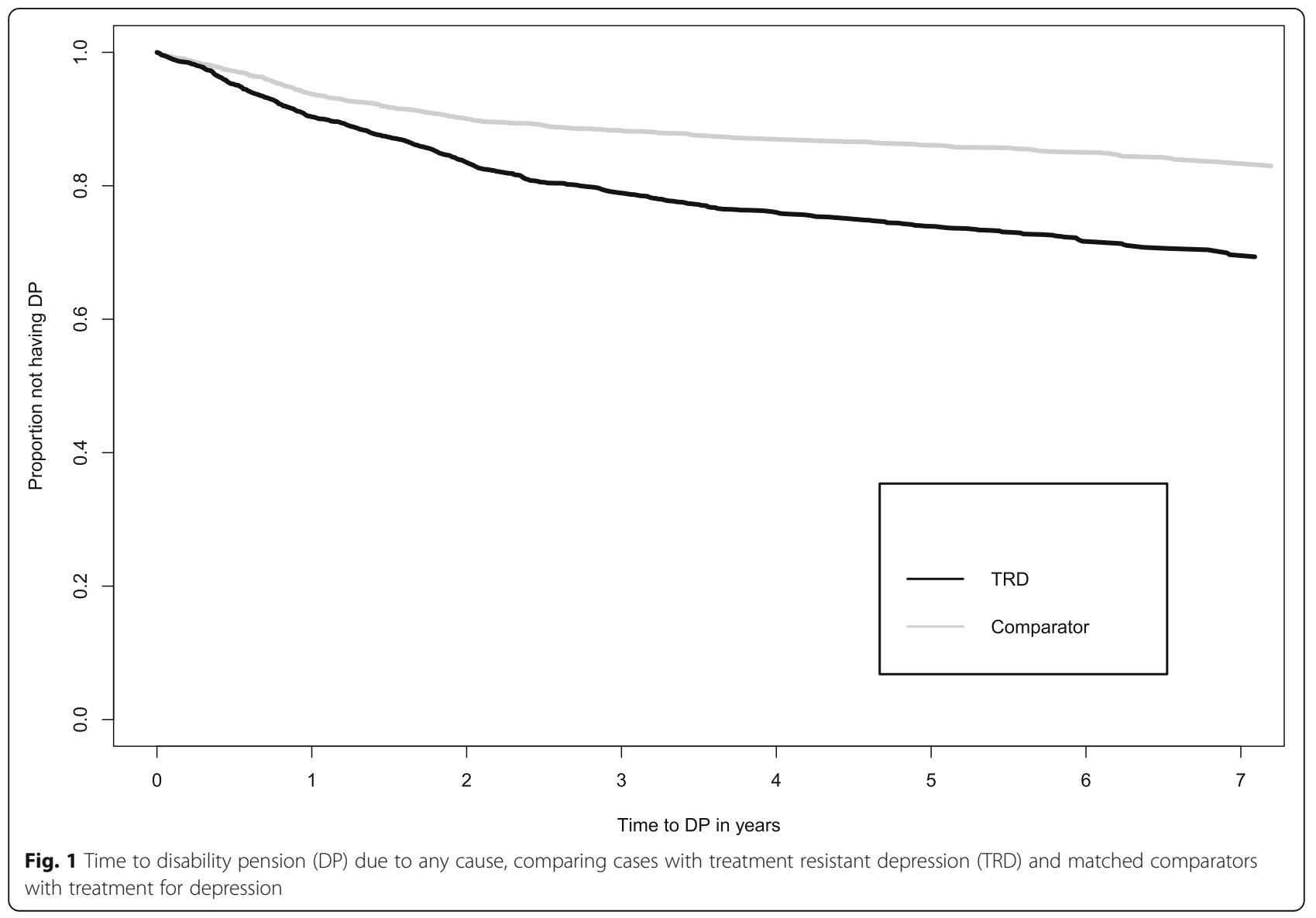


Table 3 Risk for all-cause disability pension (DP) among patients with treatment-resistant depression (TRD) vs. matched comparators with treatment for depression, stratified by matching factors. Unadjusted and adjusted hazard ratios (HR) with 95\% confidence intervals (Cl)

\begin{tabular}{|c|c|c|}
\hline & $\begin{array}{l}\text { Unadjusted HR } \\
(95 \% \mathrm{Cl})\end{array}$ & $\begin{array}{l}\text { Adjusted HR } \\
(95 \% \mathrm{Cl})^{\mathrm{a}}\end{array}$ \\
\hline All & $1.90(1.72-2.11)$ & $2.07(1.83-2.35)$ \\
\hline \multicolumn{3}{|l|}{ Sex } \\
\hline Men & $1.77(1.52-2.08)$ & $1.84(1.50-2.24)$ \\
\hline Women & $2.00(1.75-2.29)$ & $2.29(1.94-2.71)$ \\
\hline \multicolumn{3}{|l|}{ Age (years) ${ }^{b}$} \\
\hline $18-29$ & $2.31(1.90-2.82)$ & $2.50(1.97-3.16)$ \\
\hline $30-49$ & $1.80(1.54-2.10)$ & $2.05(1.68-2.50)$ \\
\hline $50-69$ & $1.61(1.32-1.97)$ & $1.85(1.39-2.46)$ \\
\hline \multicolumn{3}{|l|}{ Type of living area } \\
\hline Small municipality & $2.03(1.66-2.47)$ & $2.41(1.83-3.17)$ \\
\hline Medium-sized municipality & $1.49(1.26-1.76)$ & $1.43(1.13-1.80)$ \\
\hline Large city & $2.27(1.91-2.70)$ & $2.62(2.10-3.28)$ \\
\hline \multicolumn{3}{|c|}{ History of depression diagnosis ${ }^{\complement}$} \\
\hline Yes & $1.78(1.39-2.28)$ & $2.12(1.50-2.99)$ \\
\hline No & $1.93(1.72-2.16)$ & $2.13(1.85-2.45)$ \\
\hline \multicolumn{3}{|c|}{$\begin{array}{l}\text { adjusted for attained educational level, marital/ parental status, country of } \\
\text { birth, long-term sickness absence, unemployment, anxiety disorders, } \\
\text { personality disorders, and other psychiatric disorders, substance use disorder, } \\
\text { history of self-harm/suicide attempt and Charlson's Comorbidity index } \\
\text { bAt time of first antidepressant prescription } \\
\text { GCD-10 diagnosis F32-F33 }\end{array}$} \\
\hline
\end{tabular}

female, of older age, unemployed, born outside Europe and having a history of personality disorder were risk factors for DP.

\section{Association between TRD and DP}

Results from the present study illustrate that patients fulfilling our definition of TRD - i.e. patients initiating a third treatment trial for depression - had a substantial risk increase for DP compared with patients with an ongoing first or second treatment. A possible confounding mechanism behind the association between TRD and DP is that work disability itself is a risk factor for depression, and patients with work disability may receive more active treatment interventions from clinicians compared to those with no work disability which would lead to easier fulfilling our definition of TRD [41]. However, results were still significant after adjustment for previous long-term sickness absence. Likewise, patients actively seeking DP may have more health care contacts and hence more opportunities for treatment trials introducing follow-up bias; the matching criteria of active treatment among the comparators was meant to address this issue.
The relationship between depression, TRD and DP is bound to be complex. Comorbidity of somatic and psychiatric disorders may induce depression and treatment resistance, as well as elevate the risk for DP [42]. Depression and the risk for TRD may both be related to personality traits such as negative valence and neuroticism [43], which in turn may be independent risk factors for disability pension [44]. Factors associated with both TRD and DP may contribute to this association. Patients with TRD are more likely to spend more time in a depressed state, which contributes to the risk for DP from depression [28]. In addition, patients defined with TRD may actually be experiencing so called "pseudo-resistance" due to undiagnosed psychotic or bipolar disorders, which carry a high risk for DP [45-47].

The Kaplan-Meier curves showed the highest deviance in granted DP between TRD and comparators patients during 500-1000 days after start of follow-up and the difference remained to the end of follow-up. This finding may be of importance as it shows that the TRD definition divides patients into different risk groups years before DP is granted, which is consistent with this decision process often taking several years.

A large proportion of patients with TRD, and to a lesser degree, comparators, were granted DP during follow-up, and most had been neither on long-term sick leave (13\%) nor unemployed (25\%) at antidepressant treatment initiation (although numbers were markedly higher than the approximately $6 \%$ estimated for both measures in the general population in Sweden $[48,49])$. This may further illustrate the potential impact of both MDD and TRD on risk for DP, but should also be viewed in light of a significant proportion of the cohort being < 30 years of age, and many in this age group could be in the educational system or work apprenticeship, and hence less included in these social insurance systems.

\section{Risk factors for DP among patients with TRD}

Among the patients with TRD, women were at higher risk for DP than men, corresponding to the known elevated risk for DP among women in general [50]. As women are also at higher risk for both depression and for subsequent TRD [51], further investigation of these mechanisms seems highly prioritized considering that there are inequities regarding both mental health efforts [52] and disability pension risk [53] towards women.

Risk for DP increased with age, also in line with the existing literature [54]. Being born outside of Europe is another established risk factor, which may be attributed to adjustment difficulties to a new societal context and labour market and perhaps cultural differences regarding acceptance of mental distress and occupational function [54]. Regarding socio-economic status, lower attained 
Table 4 Risk factors for disability pension (DP) among patients with treatment-resistant depression (TRD). Unadjusted and adjusted hazard ratios (HR) with 95\% confidence intervals (Cl) for all-cause DP

\begin{tabular}{lll}
\hline & $\begin{array}{l}\text { Unadjusted HR } \\
(95 \% \mathrm{Cl})\end{array}$ & $\begin{array}{l}\text { Adjusted HR } \\
(95 \% \mathrm{Cl})^{\mathbf{a}}\end{array}$ \\
\hline $\begin{array}{lll}\text { Sex } \\
\text { Men }\end{array}$ & Ref & Ref \\
Women & $1.11(0.95-1.28)$ & $\mathbf{1 . 2 1}(\mathbf{1 . 0 3 - 1 . 4 1 )}$ \\
$\begin{array}{l}\text { Age (years) } \\
\text { 18-29 }\end{array}$ & Ref & Ref \\
$30-49$ & $1.28(1.08-1.52)$ & $\mathbf{1 . 3 2}(\mathbf{1 . 0 5 - 1 . 6 6 )}$ \\
$50-69$ & $1.63(1.34-1.98)$ & $\mathbf{1 . 7 0} \mathbf{( 1 . 3 2 - 2 . 1 9 )}$
\end{tabular}

Size of living area

\section{Small municipality \\ Medium-sized municipality}

Large city

Attained education level $^{\mathbf{b}}$
$\leq 9$ years
$10-12$ years
$\geq 13$ years

Family situation $^{\mathbf{b}}$

Married, living without children
Married, living with children
Unmarried, living without children
Unmarried, living with children
$\leq 20$ years, living with parent/-s

\section{Country of birth}

Sweden

Other European country

Other than Europe

Long-term sickness absence ${ }^{c}$

$\begin{array}{lll}\text { No } & \text { Ref } & \text { Ref } \\ \text { Yes } & 3.17(2.70-3.72) & \mathbf{3 . 2 6}(\mathbf{2 . 7 4 - 3 . 8 7 )} \\ \text { Unemployment }^{d} & & \\ \text { None } & \text { Ref } & \text { Ref } \\ 1-179 \text { days } & 1.04(0.87-1.25) & \mathbf{1 . 2 4}(\mathbf{1 . 0 3}-\mathbf{1 . 5 0}) \\ \geq 180 \text { days } & 1.11(0.82-1.50) & 1.24(0.91-1.69)\end{array}$

\section{History of depression}

No

Yes

History of anxiety disorder ${ }^{\mathrm{e}}$

$\begin{array}{lll}\text { No } & \text { Ref } & \text { Ref } \\ \text { Yes } & 0.92(0.76-1.12) & 0.91(0.75-1.12)\end{array}$

History of personality disorder

$\begin{array}{lll}\text { No } & \text { Ref } & \text { Ref } \\ \text { Yes } & 1.46(0.99-2.16) & \mathbf{1 . 5 8}(\mathbf{1 . 0 5 - 2 . 3 8 )}\end{array}$

Table 4 Risk factors for disability pension (DP) among patients with treatment-resistant depression (TRD). Unadjusted and adjusted hazard ratios (HR) with 95\% confidence intervals (CI) for all-cause DP (Continued)

\begin{tabular}{lll}
\hline & $\begin{array}{l}\text { Unadjusted HR } \\
(95 \% \mathrm{Cl})\end{array}$ & $\begin{array}{l}\text { Adjusted HR } \\
(95 \% \mathrm{Cl})^{\mathbf{a}}\end{array}$ \\
\hline $\begin{array}{ll}\text { Substance use disorder } \\
\text { None }\end{array}$ & Ref & Ref \\
Alcohol only & $0.73(0.47-1.12)$ & $0.74(0.47-1.15)$ \\
Other/mixed & $0.82(0.56-1.21)$ & $0.72(0.48-1.07)$
\end{tabular}

History of other psychiatric disorder ${ }^{\mathrm{e}}$

$$
\begin{array}{lll}
\text { No } & \text { Ref } & \text { Ref } \\
\text { Yes } & 1.12(0.81-1.54) & 1.26(0.90-1.78)
\end{array}
$$

History of self harm/suicide attempt $\mathrm{e}^{\mathrm{e}}$

\begin{tabular}{cll} 
No & Ref & Ref \\
Yes & $1.15(0.86-1.55)$ & $1.19(0.87-1.63)$ \\
CCI $^{5,6}$ & & \\
0 & Ref & Ref \\
1 & $1.60(1.18-2.17)$ & $\mathbf{1 . 4 1}(\mathbf{1 . 0 3 - 1 . 9 2 )}$ \\
$\geq 2$ & $1.34(0.69-2.59)$ & $0.87(0.45-1.69)$ \\
\hline
\end{tabular}

${ }^{\text {a Adjusted for all covariates }}$

${ }^{\mathrm{b}}$ At time of first antidepressant prescription

c > 90 net days in the 365 days preceding first antidepressant prescription

dIn the year preceding first antidepressant prescription

eWithin 5 years before first antidepressant prescription

fCharlson's comorbidity index [40]

education level did not increase risk for DP significantly compared to higher in this study, in contrast with existing literature on the impact of education in general [55]. This may reflect that the occupational disability due to TRD may be equally prominent among individuals normally at lower risk for DP such as academics. We did not include income level as a socio-economic measure in the present study, which would an interesting factor to investigate to see if the impact of TRD also attenuates the known association between low income and TRD [54]. Regarding family situation, patients in single households with children surprisingly had lower risk for DP than married/cohabitants without children. This may be related to that the substantial drop in income after DP may be a motivation factor to stay in employment as long as necessary in a perhaps already stressed economic situation. Patients $<20$ years living at home had highest risk for DP in the adjusted, but not crude, analysis, which may be related to the granting of temporary DP discussed above.

Regarding clinical risk factors, somewhat unexpectedly a history of depressive episodes did not increase the risk for DP. Recurrent depression is a known risk factor for TRD [56], but if results here are to believed, it is the TRD, not recurrence, that has an impact on risk for DP although it has been previously demonstrated that time 
spent in depression may increase risk for DP [28]. A history of suicide attempts/self-harm may be related to both severity of depression and risk for TRD, but did likewise not emerge significant in this study [16].

History of SUD and anxiety disorders did not affect risk for DP among the patients in this study. While these comorbidities may increase risk for DP in themselves, there has been little additional effect demonstrated when present as comorbid conditions in depression [28]. Personality disorders and somatic comorbidity, however, have been reported to increase risk for DP in patients with depression and emerged as significant risk factors in this study $[28,57]$.

\section{Strengths and weaknesses}

Strengths of this study include the use of nationwide Swedish registers of high quality and coverage, allowing adjustment for clinical and sociodemographic covariates, a relatively long follow-up time with no loss of followup. The matching requirement of ongoing treatment in the comparator cohort lowered the risk for follow-up bias. Also, we included only patients with a depression diagnosis from specialized healthcare, increasing the validity of the diagnosis.

Weaknesses of this study are also, however, related to the register-based setting. The definition of TRD used here is a proxy model, with unknown validity in comparison to clinical evaluation although similar models have been used in various data sources previously in Sweden and other countries [17, 29, 33] . Although clinical accuracy of the NPR is generally high, the diagnosis of depression has not yet not been validated, and validation of the corresponding register in the similar country of Denmark showed moderate precision [58]. Clinical data on severity of depression was not available. Reasons for continuing or discontinuing treatment are unknown, and may be related to adverse reactions, improvement of symptoms or loss to clinical follow-up.

\section{Implications}

While this study is set within the Swedish health care and social insurance system, the results should be generalizable to other countries with similar health care and social insurance regulations. In Sweden, DP is granted when the work capacity loss is considered longterm (for patients < 30 years old) or permanent by both the physician and by the Social Insurance Agency, which should equal the highest level of work disability. Considering the high prevalence of both depression, and eventual high rates of TRD among those initiating treatment for depression, this may infer vast economic consequences for the society, as well as a large, long-term or permanent income loss for the patient. Results from this study highlight the need for measures that prevent or improve TRD status to avoid these costs, as well as for rehabilitating measures to preserve or improve occupational functioning among patients with TRD. The fact that over a third of patients in this study were under thirty years of age and that these had the highest risk for DP compared to other depressed patients may be of special concern, especially in the context of the often early age of onset of depression [59] and that depression onset in younger age has been demonstrated to increase risk for TRD [60].

\section{Conclusion}

Patients with TRD are at higher risk for being granted DP than other patients treated for depression. Prevention of TRD status and contributing mechanisms may decrease risk for DP. The costs for society and the individual due to TRD-related work disability should be further investigated.

\section{Supplementary information}

Supplementary information accompanies this paper at https://doi.org/10. 1186/s12888-020-02642-9.

Additional file 1: Supplementary Table 1. Frequencies of persons granted with disability pension (DP) due to specific mental and behavioural disorders, compared between persons with treatment resistant depression (TRD) and comparators.

\section{Abbreviations \\ aHR: Adjusted hazard ratio; ATC: Anatomical Therapeutic Chemical; Cl: Confidence interval; DP: Disability pension; ECT: Electroconvulsive therapy; ICD-10: International Classification of Diseases, 10th version; MDD: Major depressive disorder; MiDAS: Micro-Data for Analyses of Social insurance; NPR: National Patient Register; OCD: Obsessive compulsive disorder; rTMS: Repetitive transcranial magnetic stimulation; STAR*D: Sequenced Treatment Alternatives to Relieve Depression; SUD: Substance use disorders; TPR: Total Population Register; TRD: Treatment resistant depression}

\section{Acknowledgements}

Not applicable.

\section{Authors' contributions}

Data holder: EMR. Study conceptualization: HT, JR, AT, LB, JT, EMR and PB. Statistical analysis: HT and LB. Data interpretation: HT, JR, AT, LB, JT, EMR and PB. Manuscript writing: HT, JR, AT, LB, JT, EMR and PB. Manuscript editing and approval: HT, JR, AT, LB, JT, AD, EMR and PB.

\section{Funding}

This project was funded through grants from the Söderström-Königska Foundation (grant no SLS-759771) and the Thuring Foundation (grant no 2017-00302), as well as through the public-private real-world evidence collaboration between Karolinska Institutet and Janssen Pharmaceuticals (contract: 5-63/2015). The funding agencies had no role in the design of the study, in collection, analysis, and interpretation of data, or in writing the manuscript. Open access funding provided by Karolinska Institute.

\section{Availability of data and materials}

The data that support the findings of this study are available from the Swedish government agencies National Board of Health and Welfare, and Statistics Sweden, but restrictions apply to the availability of these data, which were used under license for the current study and so are not publicly available. 


\section{Ethics approval and consent to participate}

The study was approved by the regional ethical review board in Stockholm (no 2007/762-31). Consent from participants was not deemed necessary by the board due to data already being collected and available in governmental registers, as well as being fully anonymized so no specific individual could be identified.

\section{Consent for publication}

Not applicable.

\section{Competing interests}

$J R$, LB, and PB are affiliated to or employees at CPE which receives grants from several entities (pharmaceutical companies, regulatory authorities, contract research organizations) for the performance of drug safety and drug utilization studies. AT, HT and JT have participated in research projects funded by Janssen and Eli Lilly with grants paid to the research institution. $A D$ is an employee and stockholder of Janssen Inc.

\section{Author details}

${ }^{1}$ Department of Clinical Neuroscience, Division of Insurance Medicine, Karolinska Institutet, Stockholm, Sweden. ${ }^{2}$ Department of Forensic Psychiatry, Niuvanniemi Hospital, University of Eastern Finland, Kuopio, Finland. ${ }^{3}$ School of Pharmacy, University of Eastern Finland, Kuopio, Finland. ${ }^{4}$ Centre for Pharmacoepidemiology, Department of Medicine Solna, Karolinska Institutet, Karolinska University Hospital, Stockholm, Sweden. ${ }^{5}$ Janssen Research \& Development, LLC, Titusville, NJ, USA.

Received: 7 February 2020 Accepted: 30 April 2020

\section{Published online: 13 May 2020}

\section{References}

1. Bromet E, Andrade LH, Hwang I, Sampson NA, Alonso J, de Girolamo G, et al. Cross-national epidemiology of DSM-IV major depressive episode. BMC Med. 2011;9:90 PubMed PMID: 21791035. Pubmed Central PMCID: PMC3163615. Epub 2011/07/28. eng.

2. Kessler RC, Berglund P, Demler O, Jin R, Merikangas KR, Walters EE. Lifetime prevalence and age-of-onset distributions of DSM-IV disorders in the National Comorbidity Survey Replication. Arch Gen Psychiatry. 2005;62(6): 593-602 PubMed PMID: 15939837. Epub 2005/06/09. eng.

3. Seedat S, Scott KM, Angermeyer MC, Berglund P, Bromet EJ, Brugha TS, et al. Cross-national associations between gender and mental disorders in the World Health Organization world mental health surveys. Arch Gen Psychiatry. 2009;66(7):785-95 PubMed PMID: 19581570. Pubmed Central PMCID: PMC2810067. Epub 2009/07/08. eng.

4. American Psychiatric Association. Diagnostic and statistical manual of mental disorders $\left(\mathrm{DSM}-5^{\oplus}\right)$ : American Psychiatric Publishing; Arlington, VA; 2013.

5. Patten SB, Williams JV, Lavorato DH, Modgill G, Jette N, Eliasziw M. Major depression as a risk factor for chronic disease incidence: longitudinal analyses in a general population cohort. Gen Hosp Psychiatry. 2008;30(5): 407-13 PubMed PMID: 18774423. Epub 2008/09/09. eng.

6. Rhebergen D, Beekman AT, de Graaf R, Nolen WA, Spijker J, Hoogendijk WJ, et al. Trajectories of recovery of social and physical functioning in major depression, dysthymic disorder and double depression: a 3-year follow-up. J Affect Disord. 2010;124(1-2):148-56 PubMed PMID: 19945171. Epub 2009/ 12/01. eng.

7. Machado MO, Veronese N, Sanches M, Stubbs B, Koyanagi A, Thompson T, et al. The association of depression and all-cause and cause-specific mortality: an umbrella review of systematic reviews and meta-analyses. BMC Med. 2018;16(1):112.

8. Kleine-Budde K, Müller R, Kawohl W, Bramesfeld A, Moock J, Rössler W. The cost of depression - a cost analysis from a large database. J Affect Disord. 20132013/05/01/;147(1):137-43.

9. Global Burden of Disease Study 2013 Collaborators. Global, regional, and national incidence, prevalence, and years lived with disability for 301 acute and chronic diseases and injuries in 188 countries, 1990-2013: a systematic analysis for the global burden of disease study 2013. Lancet (London, England). 2015;386(9995):743-800 PubMed PMID: 26063472. Pubmed Central PMCID: PMC4561509. Epub 2015/06/13. eng.

10. McKnight PE, Kashdan TB. The importance of functional impairment to mental health outcomes: a case for reassessing our goals in depression treatment research. Clin Psychol Rev. 2009;29(3):243-59 PubMed PMID: 19269076. Pubmed Central PMCID: PMC2814224. Epub 2009/03/10. eng.

11. Swedish Social Insurance Agency. Social Insurance in Figures 2017. Stockholm; 2017..

12. Stattin M. Retirement on grounds of ill health. Occup Environ Med. 2005; 62(2):135-40 PubMed PMID: 15657199. Pubmed Central PMCID: PMC1740964. Epub 2005/01/20. eng.

13. Amiri S, Behnezhad S. Depression and risk of disability pension: a systematic review and meta-analysis. Int J Psychiatry Med. 2019:91217419837412 PubMed PMID: 31060410. Epub 2019/05/08. eng..

14. Conway CR, George MS, Sackeim HA. Toward an evidence-based, operational definition of treatment-resistant depression: when enough is enough. JAMA Psychiat. 2017;74(1):9-10 PubMed PMID: 27784055.

15. Mclntyre RS, Filteau MJ, Martin L, Patry S, Carvalho A, Cha DS, et al. Treatment-resistant depression: definitions, review of the evidence, and algorithmic approach. J Affect Disord. 2014;156:1-7 PubMed PMID: 24314926.

16. Kautzky A, Dold M, Bartova L, Spies M, Kranz GS, Souery D, et al. Clinical factors predicting treatment resistant depression: affirmative results from the European multicenter study. Acta Psychiatr Scand. 2019;139(1):78-88 PubMed PMID: 30291625. Epub 2018/10/07. eng.

17. Reutfors J, Andersson TM, Brenner P, Brandt L, DiBernardo A, Li G, et al. Mortality in treatment-resistant unipolar depression: a register-based cohort study in Sweden. J Affect Disord. 2018;238:674-9 PubMed PMID: 29966932. Epub 2018/07/04. eng.

18. Balestri M, Calati R, Souery D, Kautzky A, Kasper S, Montgomery S, et al. Socio-demographic and clinical predictors of treatment resistant depression: a prospective European multicenter study. J Affect Disord. 2016;189:224-32 PubMed PMID: 26451508.

19. Greden JF. The burden of disease for treatment-resistant depression. J Clin Psychiatry. 2001;62(Suppl 16):26-31 PubMed PMID: 11480881. Epub 2001/ 08/02. eng.

20. Rytsala HJ, Melartin TK, Leskela US, Lestela-Mielonen PS, Sokero TP, Isometsa ET. Determinants of functional disability and social adjustment in major depressive disorder: a prospective study. J Nerv Ment Dis. 2006;194(8):570-6 PubMed PMID: 16909064. Epub 2006/08/16. eng.

21. Riihimaki $K$, Vuorilehto $M$, Isometsa E. A 5-year prospective study of predictors for functional and work disability among primary care patients with depressive disorders. Eur Psychiatry. 2015;30(1):51-7 PubMed PMID: 24721280. Epub 2014/04/12. eng.

22. Koopmans PC, Roelen CA, Groothoff JW. Sickness absence due to depressive symptoms. Int Arch Occup Environ Health. 2008;81(6):711-9 PubMed PMID: 17849142. Pubmed Central PMCID: PMC2254471. Epub 2007/09/13. eng.

23. Trivedi MH, Corey-Lisle PK, Guo Z, Lennox RD, Pikalov A, Kim E. Remission, response without remission, and nonresponse in major depressive disorder: impact on functioning. Int Clin Psychopharmacol. 2009;24(3):133-8 PubMed PMID: 19318972. Epub 2009/03/26. eng.

24. Fostick L, Silberman A, Beckman M, Spivak B, Amital D. The economic impact of depression: resistance or severity? Eur Neuropsychopharmacol. 2010;20(10):671-5 PubMed PMID: 20624674. Epub 2010/07/14. eng.

25. DiBernardo A, Lin X, Zhang Q, Xiang J, Lu L, Jamieson C, et al. Humanistic outcomes in treatment resistant depression: a secondary analysis of the STAR*D study. BMC Psychiatry. 2018;18(1):352 PubMed PMID: 30373547. Pubmed Central PMCID: PMC6206859. Epub 2018/10/31. eng.

26. Fekadu A, Wooderson SC, Markopoulo K, Donaldson C, Papadopoulos A, Cleare AJ. What happens to patients with treatment-resistant depression? A systematic review of medium to long term outcome studies. J Affect Disord. 2009;116(1-2):4-11 PubMed PMID: 19007996. Epub 2008/11/15. eng.

27. Mittendorfer-Rutz E, Harkanen T, Tiihonen J, Haukka J. Association of sociodemographic factors, sick-leave and health care patterns with the risk of being granted a disability pension among psychiatric outpatients with depression. PLoS One. 2014;9(6):e99869 PubMed PMID: 24963812. Pubmed Central PMCID: PMC4070900. Epub 2014/06/26. eng.

28. Holma IAK, Holma KM, Melartin TK, Rytsälä HJ, Isometsä ET. A 5-year prospective study of predictors for disability pension among patients with major depressive disorder. Acta Psychiatr Scand. 2012;125(4):325-34.

29. Olchanski N, Mclnnis Myers M, Halseth M, Cyr PL, Bockstedt L, Goss TF, et al. The economic burden of treatment-resistant depression. Clin Ther. 2013; 35(4):512-22 PubMed PMID: 23490291. 
30. Corey-Lisle PK, Birnbaum HG, Greenberg PE, Marynchenko MB, Claxton AJ. Identification of a claims data "signature" and economic consequences for treatment-resistant depression. J Clin Psychiatry. 2002;63(8):717-26 PubMed PMID: 12197453. Epub 2002/08/29. eng.

31. Gibson TB, Jing Y, Smith Carls G, Kim E, Bagalman JE, Burton WN, et al. Cost burden of treatment resistance in patients with depression. Am J Manag Care. 2010;16(5):370-7 PubMed PMID: 20469957. Epub 2010/05/18. eng.

32. Kubitz N, Mehra M, Potluri RC, Garg N, Cossrow N. Characterization of treatment resistant depression episodes in a cohort of patients from a US commercial claims database. PLoS One. 2013;8(10):e76882 PubMed PMID: 24204694. Pubmed Central PMCID: PMC3799999.

33. Byford S, Barrett B, Despiegel N, Wade A. Impact of treatment success on health service use and cost in depression: longitudinal database analysis. PharmacoEconomics. 2011;29(2):157-70 PubMed PMID: 21142289. Epub 2010/12/15. eng

34. Fife D, Feng Y, Wang MY, Chang CJ, Liu CY, Juang HT, et al. Epidemiology of pharmaceutically treated depression and treatment resistant depression in Taiwan. Psychiatry Res. 2017;252:277-83 PubMed PMID: 28288438. Epub 2017/03/14. eng.

35. Wettermark B, Hammar N, Fored CM, Leimanis A, Otterblad Olausson P, Bergman $U$, et al. The new Swedish prescribed drug register-opportunities for pharmacoepidemiological research and experience from the first six months. Pharmacoepidemiol Drug Saf. 2007;16(7):72635 PubMed PMID: 16897791.

36. Dold M, Kasper S. Evidence-based pharmacotherapy of treatment-resistant unipolar depression. Int J Psychiatry Clin Pract. 2017;21(1):13-23 PubMed PMID: 27848269. Epub 2016/11/17. eng

37. Ludvigsson JF, Andersson E, Ekbom A, Feychting M, Kim JL, Reuterwall C, et al. External review and validation of the Swedish national inpatient register. BMC Public Health. 2011;11:450 PubMed PMID: 21658213. Pubmed Central PMCID: PMC3142234. Epub 2011/06/11. eng.

38. Ludvigsson JF, Almqvist C, Bonamy AK, Ljung R, Michaelsson K, Neovius M, et al. Registers of the Swedish total population and their use in medical research. Eur J Epidemiol. 2016;31(2):125-36 PubMed PMID: 26769609. Epub 2016/01/16. eng.

39. Swedish Social Security Agency; MiDAS, sickness benefits and rehabilitation benefits. v1.02 (2014). Available at http://www.forsakringskassan.se.

40. Charlson ME, Pompei P, Ales KL, Mackenzie CR. A new method of classifying prognostic comorbidity in longitudinal studies: development and validation. J Chronic Dis. 1987:40(5):373-83 PubMed PMID: 3558716. Epub 1987/01/01. eng.

41. Wang M, Alexanderson K, Runeson B, Mittendorfer-Rutz E. Morbidity and suicide mortality following sick leave in relation to changes of social insurance regulations in Sweden. Eur J Public Health. 2016;26(6):1061-9 PubMed PMID: 27418585. Epub 2016/07/16. eng.

42. Knudsen AK, Overland S, Aakvaag HF, Harvey SB, Hotopf M, Mykletun A. Common mental disorders and disability pension award: seven year followup of the HUSK study. J Psychosom Res. 2010;69(1):59-67 PubMed PMID: 20630264. Epub 2010/07/16. eng.

43. Takahashi M, Shirayama Y, Muneoka K, Suzuki M, Sato K, Hashimoto K. Personality traits as risk factors for treatment-resistant depression. PLoS One. 2013;8(5):e63756 PubMed PMID: 23717477. Pubmed Central PMCID: PMC3661718. Epub 2013/05/30. eng.

44. Ropponen A, Svedberg P, Huunan-Seppälä A, Koskenvuo K, Koskenvuo $M$, Alexanderson $K$, et al. Personality traits and life dissatisfaction as risk factors for disability pension due to low back diagnoses: a 30-year longitudinal cohort study of Finnish twins. J Psychosom Res. 20122012/ 10/01/;73(4):289-94.

45. Fornaro M, Giosue P. Current nosology of treatment resistant depression: a controversy resistant to revision. Clin Pract Epidemiol Mental Health. 2010;6: 20-4 PubMed PMID: 20563286. Pubmed Central PMCID: PMC2887642. Epub 2010/06/22. eng.

46. Ringdal PR, Haug K, Gunnar MJ. Long-term sickness absence and disability pension with psychiatric diagnoses: a population-based cohort study AU Gjesdal, Sturla. Nord J Psychiatry. 2008 2008/01/01;62(4):294-301.

47. Schoeyen HK, Birkenaes AB, Vaaler AE, Auestad BH, Malt UF, Andreassen OA, et al. Bipolar disorder patients have similar levels of education but lower socio-economic status than the general population. J Affect Disord. 20112011/03/01/;129(1):68-74.

48. Wang M, Alexanderson K, Runeson B, Head J, Melchior M, Perski A, et al. Are all-cause and diagnosis-specific sickness absence, and sick-leave duration risk indicators for suicidal behaviour? A nationwide register-based cohort study of 4.9 million inhabitants of Sweden. Occup Environ Med. 2014;71(1): 12-20 PubMed PMID: 24142975. Epub 2013/10/22. eng.

49. Statistics Sweden. Labour forces surveys [May 9th]; 2019. Available from: https://www.scb.se/en/finding-statistics/statistics-by-subject-area/labourmarket/labour-force-surveys/labour-force-surveys-lfs/.

50. Albertsen K, Lund T, Christensen KB, Kristensen TS, Villadsen E. Predictors of disability pension over a 10-year period for men and women. Scand J Public Health. 2007;35(1):78-85 PubMed PMID: 17366091. Epub 2007/03/17. eng.

51. Gronemann FH, Jorgensen MB, Nordentoft M, Andersen PK, Osler M. Sociodemographic and clinical risk factors of treatment-resistant depression: a Danish population-based cohort study. J Affect Disord. 2020;261:221-9 PubMed PMID: 31655377. Epub 2019/10/28. eng.

52. Yu S. Uncovering the hidden impacts of inequality on mental health: a global study. Transl Psychiatry. 2018;8(1):98 PubMed PMID: 29777100. eng.

53. Haukenes I, Löve J, Hensing G, Knudsen AK, Øverland S, Vahtera J, et al. Inequity in disability pension: an intersectional analysis of the coconstitution of gender, education and age. The Hordaland health study. Crit Public Health. 20192019/05/27;29(3):302-13.

54. Karlsson NE, Carstensen JM, Gjesdal S, Alexanderson KA. Risk factors for disability pension in a population-based cohort of men and women on long-term sick leave in Sweden. Eur J Public Health. 2008;18(3):224-31 PubMed PMID: 18245150. Epub 2008/02/05. eng.

55. Bruusgaard D, Smeby L, Claussen B. Education and disability pension: a stronger association than previously found. Scand J Public Health. 2010; 38(7):686-90 PubMed PMID: 20709890. Epub 2010/08/17. eng.

56. Bennabi D, Aouizerate B, El-Hage W, Doumy O, Moliere F, Courtet P, et al. Risk factors for treatment resistance in unipolar depression: a systematic review. J Affect Disord. 2015;171:137-41 PubMed PMID: 25305428.

57. Østby KA, Czajkowski N, Knudsen GP, Ystrom E, Gjerde LC, Kendler KS, et al. Personality disorders are important risk factors for disability pensioning. Soc Psychiatry Psychiatr Epidemiol. 2014;49(12):2003-11.

58. Bock C, Bukh JD, Vinberg M, Gether U, Kessing LV. Validity of the diagnosis of a single depressive episode in a case register. Clin Pract Epidemiol Ment Health. 2009;5(1):4

59. Otte C, Gold SM, Penninx BW, Pariante CM, Etkin A, Fava M, et al. Major depressive disorder. Nat Rev Dis Primers. 2016;2:16065 PubMed PMID: 27629598

60. Souery D, Oswald P, Massat I, Bailer U, Bollen J, Demyttenaere K, et al. Clinical factors associated with treatment resistance in major depressive disorder: results from a European multicenter study. J Clin Psychiatry. 2007; 68(7):1062-70 PubMed PMID: 17685743. Epub 2007/08/10. eng

\section{Publisher's Note}

Springer Nature remains neutral with regard to jurisdictional claims in published maps and institutional affiliations.

Ready to submit your research? Choose BMC and benefit from:

- fast, convenient online submission

- thorough peer review by experienced researchers in your field

- rapid publication on acceptance

- support for research data, including large and complex data types

- gold Open Access which fosters wider collaboration and increased citations

- maximum visibility for your research: over $100 \mathrm{M}$ website views per year

At $\mathrm{BMC}$, research is always in progress.

Learn more biomedcentral.com/submission 